See discussions, stats, and author profiles for this publication at: https://www.researchgate.net/publication/344033137

\title{
Mechanism design for efficient allocation of electric vehicles to charging stations
}

Conference Paper · September 2020

DOI: $10.1145 / 3411408.3411434$

CITATIONS

0

5 authors, including:

Emmanouil S. Rigas

Aristotle University of Thessaloniki

23 PUBLICATIONS 221 CITATIONS

SEE PROFILE

Sebastian Stein

4. University of Southampton

91 PUBLICATIONS 1,001 CITATIONS

SEE PROFILE

Some of the authors of this publication are also working on these related projects:

Project Al4EU - Europe's Al-on-Demand Platform - Explainable Al View project

Project The logic perspective of computation View project
READS

12

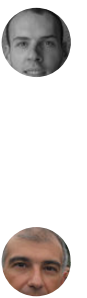

Enrico H. Gerding

University of Southampton

194 PUBLICATIONS 2,263 CITATIONS

SEE PROFILE

Nick Bassiliades

Aristotle University of Thessaloniki

262 PUBLICATIONS 3,117 CITATIONS

SEE PROFILE 


\section{Mechanism design for efficient allocation of electric vehicles to charging stations}

\author{
Emmanouil S. Rigas \\ Department of Informatics, Aristotle \\ University of Thessaloniki \\ erigas@csd.auth.gr \\ Thessaloniki, Greece
}

\author{
Enrico Gerding \\ Electronics and Computer Science, \\ University of Southampton \\ eg@ecs.soton.ac.uk \\ Southampton, UK
}

\author{
Sebastian Stein \\ Electronics and Computer Science, \\ University of Southampton \\ ss2@ecs.soton.ac.uk \\ Southampton, UK
}

\author{
Sarvapali D. Ramchurn \\ Electronics and Computer Science, \\ University of Southampton \\ sdr1@soton.ac.uk \\ Southampton, UK
}

\author{
Nick Bassiliades \\ Department of Informatics, Aristotle \\ University of Thessaloniki \\ nbassili@csd.auth.gr \\ Thessaloniki, Greece
}

\begin{abstract}
The electrification of transport can significantly reduce $\mathrm{CO}_{2}$ emissions and their negative impact on the environment. In this paper, we study the problem of allocating Electric Vehicles (EVs) to charging stations and scheduling their charging. We develop an offline solution that treats EV users as self-interested agents that aim to maximise their profit and minimise the impact on their schedule. We formulate the problem of the optimal EV to charging station allocation as a Mixed Integer Programming (MIP) one and we propose two pricing mechanisms: A fixed-price one, and another that is based on the well known Vickrey-Clark-Groves (VCG) mechanism. We observe that the VCG mechanism services on average $1.5 \%$ more EVs than the fixed-price one. In addition, when the stations get congested, VCG leads to higher prices for the EVs and higher profit for the stations, but lower utility for the EVs. However, the VCG mechanism guarantees truthful reporting of the EVs' preferences.
\end{abstract}

\section{CCS CONCEPTS}

- Computing methodologies $\rightarrow$ Planning and scheduling; Multiagent systems; Search methodologies.

\section{KEYWORDS}

Electric vehicles, mechanism design, fixed price, vcg, scheduling

\section{ACM Reference Format:}

Emmanouil S. Rigas, Enrico Gerding, Sebastian Stein, Sarvapali D. Ramchurn, and Nick Bassiliades. 2020. Mechanism design for efficient allocation of electric vehicles to charging stations. In 11th Hellenic Conference on Artificial Intelligence (SETN 2020), September 2-4, 2020, Athens, Greece. ACM, New York, NY, USA, 6 pages. https://doi.org/10.1145/3411408.3411434

Permission to make digital or hard copies of all or part of this work for personal or classroom use is granted without fee provided that copies are not made or distributed for profit or commercial advantage and that copies bear this notice and the full citation on the first page. Copyrights for components of this work owned by others than ACM must be honored. Abstracting with credit is permitted. To copy otherwise, or republish, to post on servers or to redistribute to lists, requires prior specific permission and/or a fee. Request permissions from permissions@acm.org.

SETN 2020, September 2-4, 2020, Athens, Greece

(c) 2020 Association for Computing Machinery.

ACM ISBN 978-1-4503-8878-8/20/09...\$15.00

https://doi.org/10.1145/3411408.3411434

\section{INTRODUCTION}

The increasingly negative impact of climate change on society has forced several countries to instigate national plans to reduce carbon emissions [1]. The electrification of transport is one of the main pathways to significantly reduce $\mathrm{CO}_{2}$ emissions. However, the successful introduction of EVs into the market lies upon the acceptance of the new type of vehicle by the customers. Currently, three main problems prevent the spread of EVs: 1) the relatively small range, 2) the long charging times and the unavailability of charging stations, and 3) the higher cost of buying an EV compared to a conventional car. Given that these limitations demand several years before they can potentially be removed, ways of making EVs attractive to customers given the current situation must be developed. For example, the coordinated charging of many EVs given the available stations and the power grid constraints, as well as the fair pricing of the electricity can soften limitations 2 and 3.

In this paper we study a municipality-based urban EV charging setting where EVs drive across a city converge to parking stations in the center and need to charge. The EVs are modelled as selfinterested agents that want to charge their battery given a set of preferences and constraints, while from the system's perspective, the maximization of EV satisfaction and the balanced distribution of them across the charging stations and within each station are the objectives. At the same time, the system needs to make a profit and be economically sustainable, but maximizing the profit is not the goal. Hence, it is crucial to leverage advances in decentralized control and mechanism design to coordinate demand and supply to mitigate the impact on the grid.

In order to allocate the EVs to charging points we propose optimal offline solutions that use market-based techniques. The EVs report their preferences (e.g., energy demand, time of arrival) a day ahead and the system selects to charge the EVs with the higher valuations given the charging station and network constraints. The valuation is a metric of how much the agents want the energy units (i.e., the maximum price that the agent would be willing to pay for an amount of energy). After the allocation has been decided, two pricing mechanisms are used. In the first one, each agent pays a fixed price for each unit of electricity, while in the second one, each 
agent pays a price calculated by the well-known Vickrey-ClarkGroves (VCG) mechanism [15], [2], [6].

In recent years, market-based techniques to schedule the charging of EVs across stations have already been applied in a number of occasions [10]. Initial work by Foster and Caramanis [3], investigate market-based control techniques in collectives of EVs for load balancing and to provide regulation services that allow renewable energy sources to be integrated. They develop a bidding strategy for an EV aggregator to operate in hour-ahead markets. Moreover, Gerding et al. [4] study a setting where agents state time windows within which they will be available to charge, and bid for units of electricity in a periodic multi-unit auction. In order to ensure truthfulness, the authors developed a mechanism that occasionally leaves units of electricity unallocated, even if there is demand for them. Ghosh and Aggarwal [5] present an online pricing mechanism where each charging station decides on the prices that each EV will charge based on the available energy and the time of the day. Tackling the more general EV charging scheduling problem, Liu et al [8] formulate the problem as a hierarchical mixed-variable optimization problem, considering the dependency among the station selection, the charging option at each station and the charging amount settings. They propose a Mixed-Variable Differentiate Evolution as the scheduling algorithm for the proposed scheduling scheme. Finally, Seitaridis et al. [12] propose an agent-based negotiation scheme for the distribution of EVs across a set of charging stations aiming to maximise serviced EVs and renewable energy utilisation.

In this paper, we build on the works by Rigas et al. [11] and Stein et al. [14]. In particular, [11] applies congestion pricing across nodes in a network of charging stations to incentivise EV-agents to charge in stations with low congestion. At the same time, [14] propose a mechanism for allocating electric power units to self-interested agents, aiming to maximise social welfare of the agents. In this paper, we propose a market-based EV to charging station scheduling scheme. This scheme maximizes social welfare from the side of the EVs and guarantees truthful reporting of their preferences, while it minimises charging imbalance both across the charging stations, as well as within each station. Our contributions to the state of the art are: 1) We propose an optimal centralized offline solution using Mixed Integer Programming (MIP) to solve the problem of allocating EVs to charging stations. In doing so, we take into consideration the cost of the electricity, but also an imbalance cost which represents the difference of the actual demand compared to the expected one. 2) We propose two different pricing mechanisms. 3) We experimentally evaluate our algorithms in a setting using real locations of charging stations in Athens, Greece and we show that they achieve high EV satisfaction and have good scalability (i.e., tenths of charging stations and hundreds of EVs).

The rest of the paper is structured as follows: Section 2 formally defines the problem, Section 3 presents the optimal EV to charging station allocation scheme, Section 4 presents the two pricing mechanisms, Section 5 presents the empirical evaluation and Section 6 concludes and presents ideas for future work.

\section{PROBLEM DEFINITION}

We define a set of locations $l \in L, L=L_{p} \cup L_{\bar{p}}$ that can either be charging stations (i.e., $L_{p}$ ) or not (i.e., $\left.L_{\bar{p}}\right)$. Based on this, we define a transport network as a graph $G=(E, L)$ with nodes $L$ and edges $E$, where $e \in E$ represents the roads and $l \in L$ represents the junctions of the road network. We define discrete time points $t \in T, T \subseteq \mathbb{N}$, while we denote the set of EVs as $a \in A$.

Now, $\forall l \in L_{p}$ there are a number of charging slots $s_{l} \in \mathbb{N}$. Each charging station $l$ has a charging rate, $c_{l} \in \mathbb{R}_{0}^{+}$and all charging stations have a fixed cost $\operatorname{cost}_{l}^{\text {elec }} \in \mathbb{N}$ to pay to the electricity provider for each unit of electricity. Thus, we define an allocation matrix charge $e_{a, l, t} \in\{0,1\}$ to represent $\mathrm{EV} a$ is charging at charging station $l$ at time point $t$. Moreover, each charging station has an expected demand $\operatorname{dem}_{l, t} \in \mathbb{N}$ for each time point, which is assumed to be agreed with the electricity provider in advance. A monetary penalty cost $t_{l, t}^{i m b l}$

$$
\operatorname{cost}_{l, t}^{i m b l}=\left|\sum_{a} \operatorname{charge}_{a, l, t}-\operatorname{dem}_{l, t}\right| \times \operatorname{cost}^{i m b l}
$$

is applied to the stations whenever the actual demand varies from the expected one. The term imbalance is a measure of how much the actual demand deviates from the expected one. This imbalance cost is calculated as the sum of the absolute value of the difference between the sum of EVs charging at each time point and the expected demand dem $_{a, l}$ over all charging points multiplied by a fixed cost (see Equation 1). In other words, the closer the actual demand is to the expected one, the lower this cost is and therefore the price penalty that each station will have to pay. The difference in the absolute value is the imbalance in terms of energy being used and the cost ${ }^{i m b l}$ is a fixed price the station pays to the electricity provider when the actual demand is different from the expected one. Henceforth, index $a$ refers to the EVs, $l$ to the charging stations, and $t$ to the time points.

Each EV $a$ is considered as an autonomous agent and has a type $\theta_{a}=\left\langle d_{a}, b_{a}^{\text {max }}, b_{a, t}, l_{a}^{\text {start }}, t_{a}^{\text {start }}, l_{a}^{\text {end }}, \tau_{a}^{\text {prk }}, t_{a, l}^{\text {arr }}, t_{a, l}^{\text {dep }}, b_{a}^{\text {chrg }}, v_{a}^{\text {elec }}\right\rangle$. Each $a$ has a discharge rate $d_{a} \in \mathbb{R}_{0}^{+}$, a maximum battery capacity $b_{a}^{\max } \in \mathbb{R}_{0}^{+}$, and a current battery level at time $t, b_{a, t} \in \mathbb{R}_{0}^{+}$both measured in $\mathrm{kWh}$. Moreover, each EV $a$ departs from its source location $l_{a}^{\text {start }}$ at time $t_{a}^{\text {start }} \in T$ and wants to travel to destination $l_{a}^{e n d}$ where it needs to park for time $\tau_{a}^{p r k} \in \mathbb{N}$. Given a pair of locations $\left(l_{a}^{\text {start }}, l_{a}^{\text {end }}\right)$ and the transport network $G$, the shortest

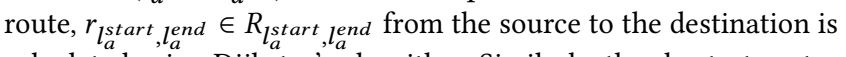
calculated using Dijkstra's algorithm. Similarly, the shortest routes $r_{l^{\prime}, l} \in R_{l^{\prime}, l}$ from the sources to all charging points $l \in L_{p}$ are calculated as well. Every route $r_{l^{\prime}, l}$ has a distance $\delta_{l^{\prime}, l}: R_{l^{\prime}, l} \rightarrow$ $\mathbb{N}$ measured in kilometers, a time to travel $\tau_{l^{\prime}, l}^{\text {drive }}: R_{l^{\prime}, l} \rightarrow \mathbb{N}$, and an amount of energy needed, $\epsilon_{l^{\prime}, l}^{\text {need }}\left(\tau_{l^{\prime}, l}^{\text {drive }}, d_{a}\right)$. Based on slot availability and on the ability of an EV to reach a charging point with its initial battery level, a set of valid charging points $\Gamma_{a, t} \subseteq L_{p}$ is defined. Every EV is available to charge between $t_{a, l}^{a r r}=t_{a}^{\text {start }}+$ $\tau_{a, l}^{d r i v e}$ and $t_{a, l}^{d e p}=t_{a, l}^{a r r}+\tau_{a}^{p r k}$. Note that $\tau_{a, l}^{d r i v e}$ is measured based on the distance to location $l$ divided by an average speed. Each vehicle also needs to charge a specific amount of energy $b_{a}^{\text {chrg }} \leq b_{a}^{\text {max }}$ 
at charging point $l$ and it also has a personal valuation $v_{a, l}$ for charging the desired amount of energy at each charging point.

Each EV has a personal valuation for charging the desired amount of electricity at a specific location.

$$
v_{a, l}= \begin{cases}\left(v_{a}^{\text {elec }}-\kappa_{a, l}^{\text {time }}\right) \times b_{a}^{c h r g}, & \text { if } b_{a, t_{a, l}^{\text {dep }}} \geq b_{a}^{c h r g} \\ 0, & \text { otherwise }\end{cases}
$$

According to Equation 2, a time cost $\kappa_{a, l}^{\text {time }}$ related to driving to the station and walking from the station to the final destination is subtracted from the valuation $v_{a}^{\text {elec }}$ for charging one unit of electricity. $v_{a}^{\text {elec }}$ and $\kappa_{a, l}^{\text {time }}$ are within the $0-1$ range. Note that $\kappa_{a, l}^{\text {time }}$ is computed similarly for all agents as it is based on the graph that is being used to represent the road network. Also note that, the agent has zero valuation for charging less than $b_{a}^{c h r g}$, and valuation $v_{a, l}$ for charging equal to or more than $b_{a}^{c h r g}$. Moreover, each agent $a$ receives utility $u_{a}$,

$$
u_{a}=v_{a, l}-p_{a}
$$

where $p_{a} \in \mathbb{R}$ is a monetary transfer from the EV to the system (i.e., the utility is a measure of satisfaction for charging the desired amount of electricity). $p_{a}$ is usually positive, as the EVs pay the charging station for the electricity. However, in case the charging of an EV leads to lower imbalance cost for the charging station, the transfer for this EV may be negative (i.e., the EV gets paid- see Section 4.2).

For an EV to be assigned to a station and charge, it has to reveal its type $\theta_{a}$ to the system. Then, the system applies an EV to charging station allocation algorithm to schedule EV charging and uses one of two proposed pricing mechanisms to calculate the prices.

\section{OFFLINE SCHEDULING OF EVS TO CHARGING POINTS}

Here, we present a formulation for optimally allocating EVs to charging points. Thus, we formulate the problem as a Mixed Integer Programming (MIP) one and we solve it optimally using IBM ILOG CPLEX 12.10. In our formulation we define two decision variables: 1) $\phi_{a, l} \in\{0,1\}$ which indicates whether an agent $a$ is serviced at charging station $l$ and 2) charge $_{a, l, t} \in\{0,1\}$ which indicates whether agent $a$ is charging at charging station $l$, at time point $t$ (at charging rate $c_{a}$ ). The objective of this formulation is to maximize Equation 4, which means to maximize the sum of the agents' valuations (i.e., charge as many agents as possible at the stations with the lower time cost) minus the cost for the electricity and minus the imbalance cost for an optimal allocation $X^{*}$ of EVs to charging points. Note that the absolute value in the objective function is linearized at run time by CPLEX. The linear program is given by:

Objective function:

$$
\begin{aligned}
F\left(X^{*}\right)=\sum_{a} \sum_{l} v_{a, l} \times \phi_{a, l}- & \sum_{a} \sum_{l} \sum_{t} \operatorname{charge}_{a, l, t} \times \\
& \operatorname{cost}_{l}^{\text {electr }}-\sum_{l} \sum_{t} \operatorname{cost}_{l, t}^{\text {imbl }}
\end{aligned}
$$

Subject to:

$$
\begin{gathered}
\sum_{l} \phi_{a, l} \leq 1, \forall a \\
b_{a}^{a r r} \geq \epsilon_{a, l}^{\text {need }}\left(r_{a, l}, d_{a}\right) \times \phi_{a, l}, \forall a, \forall l \\
\sum_{t \geq t_{a, l}^{a r r} \cap t<t_{a, l}^{\text {dep }}} \operatorname{charge}_{a, l, t} \geq\left[b_{a, l}^{\text {chrg }} / c_{l}\right] \times \phi_{a, l}, \forall a, \forall l \\
\sum_{t<t_{i, j}^{\text {arr }} \cap t \geq t_{i, j}^{\text {dep }}} \operatorname{charge}_{i, j, t}=0, \forall i, \forall j \\
\sum_{t \geq t_{a, l}^{\text {arr }} \cap t<t_{a, l}^{\text {dep }}} \operatorname{charge}_{a, l, t}+b_{a}^{\text {arr }} \leq b_{a}^{\text {max }} \\
\sum_{a} \text { charge }_{a, l, t} \leq s_{l}, \forall l \forall t
\end{gathered}
$$

We detail the key elements of this mathematical program as follows. EV $a$ will charge at most at one charging station $l$ (Eq. 5), and the initial battery level of each EV must be enough to reach the charging station selected to charge (Eq. 6). In addition, the number of time points that an EV will be charging must be greater or equal to the energy demand divided by the charging rate $c_{l}$ at station $l$ $\left(\tau_{a, l}^{c h r g}=\left[b_{a, l}^{c h r g} / c_{l}\right]\right)($ Eq. 7$)$. Moreover, for all time points before the arrival and after the departure of EV $a$ at charging station $l$, no charging must take place (Eq. 8), and after the completion of the charging, the maximum capacity of the EV's battery must not be exceeded (Eq. 9). Finally, the maximum capacity of each charging station, in terms of available chargers, must not be violated at any time (Eq. 10).

In the next Section, we present two pricing mechanisms for the electricity that each EV charges.

\section{PRICING MECHANISMS}

In this section, we present two mechanisms for calculating the price that the agents will pay for the electricity they will charge according to the scheduling as presented in the previous section. In the first mechanism, the agents are assumed to be truthful, while in the second one they may misreport their types.

\subsection{Cooperative Agents}

Initially, we assume that all agents are cooperative (i.e., they report their types $\theta_{a}$ truthfully) and we design a fixed price mechanism where the payments of the agents to the charging company are calculated based on:

$$
p_{a}^{\text {coop }}=\left(b_{a}^{\text {chrg }} \times \operatorname{cost}_{l}^{\text {elec }}\right) \times(1+i n c r)
$$

Each agent pays its energy demand multiplied by the cost of electricity for each energy unit increased by a percentage incr $\in \mathbb{R}^{+}$. This value determines the profit that the charging station will make for each unit of electricity that sells to each EV. This is actually the usual price setting mechanism in many markets, where the seller prices a product based on its cost increased by a fixed percentage. In order to calculate the incr we find the point where the mechanism becomes sustainable, i.e., stops making losses and starts making a 
small profit (see detailed description in Section 5). We will refer to this mechanism as Coop.

The allocation of the agents to charging points takes place based on the objective function (Equation 4). However, the price to pay is calculated afterwords and the valuation of the agents is not taken into consideration. Thus, the price to pay can be higher than an agent's valuation. In this case, the agent decides not to charge and receives zero utility. Thus, the agents' utilities are always equal or larger than zero and the mechanism is individually rational.

Under the assumption that all agents are truthful, they will report their true valuations and for this reason the mechanism would be incentive compatible. However, as long as an agent will not get negative utility it has an incentive to misreport its valuation. For example, assuming that the imbalance cost is equal to zero, and incr $=0.05$, if an agent has valuation $v_{a, l}=5$ but reports $v_{a, l}^{\prime}=6$, and $b_{i} * \operatorname{cost}_{l}^{\text {electr }}=4$, the optimizer will schedule this agent to charge and the price to pay will be $p_{a}^{\text {coop }}=4+4 * 0.05=4.2$. Thus, the agent will charge with the same price, but it will increase the chances of being selected instead of another agent that would report its valuation truthfully. For this reason, the mechanism is not incentive compatible.

Now, in order the mechanism to be efficient, Equation 4 must be maximized. Under the assumption that all agents are truthful, the optimization procedure leads to an allocation of EVs to charging points which maximizes this function. However, in case for some agents the price to pay is higher than their valuation, these agents will decide not to charge. Thus, some resources will remain unallocated. Also, the assumption that the agents will be truthful does not always hold. For these reasons, the mechanism is not efficient.

Finally, in the case where the actual demand is equal to the expected one, then the mechanism will make a profit as totalcost $=$ $b[i] * \operatorname{cost}^{\text {elec }}+0$ and totalRevenue $=b_{i} *\left(\cos t^{\text {elec }}+\operatorname{cost}^{\text {elec }} \times\right.$ incr $)>$ totalCost. If the demand is different than the expected one, then the budget can be either positive or negative. Only in the case where $\sum_{l} \sum_{t}\left(\operatorname{cost}_{l, t}^{i m b l}\right)=\sum_{i}\left(b_{i} \times \operatorname{cost}^{\text {elec }} \times\right.$ incr $)$ the mechanism is budget balanced. Thus, in the general case, the mechanism is not budget balanced. In the next section we present an alternative pricing mechanism which makes truthful reporting of preferences the dominant strategy for all agents.

\subsection{Strategic Agents}

In the general case, agents would try to misreport their types if they had an incentive to do so (i.e., achieve higher utility). The mechanism presented in the previous section, can easily be manipulated if some agents misreport their type (e.g., report higher valuation). Here, we present an optimal EV to charging station allocation scheme which uses the well known Vickrey-Clarke-Groves (VCG) mechanism [15] [2], [6]. The VCG mechanism is a generalization of the Vickrey auction where, in the general case, multiple agents bid for multiple goods of the same type (i.e., combinatorial auction) and the price to pay for each agent is calculated based on the harm they cause to the other agents [16]. The main characteristic of this mechanism is that it is incentive compatible, which means that no agent can benefit by declaring anything other than its true type. Therefore, this mechanism, assuming that all agents play their dominant strategies, finds the optimal allocation of the resources (i.e., electricity units) in terms of social welfare maximization and then calculates the price that each agent will pay to the mechanism.

In order to calculate the allocation of EVs to charging points, we use the MIP formulation as described in Section 3. In the end of the optimization procedure, an optimal allocation $X^{*}$ of EVs to charging points is achieved. We calculate the transfer $p_{a}^{v c g}$ (i.e., the price) that EV $a$ will pay to the mechanism for the energy charged, as follows:

$$
\begin{gathered}
p_{a}^{v c g}=\left(\sum_{e \in A}\left(v_{e, l}\left(X_{-a}^{*}\right)-\operatorname{cost}_{e}^{e l e c}\left(X_{-a}^{*}\right)-\operatorname{cost}_{e}^{i m b l}\left(X_{-a}^{*}\right)\right)\right)- \\
\left(\sum_{e \in A}\left(v_{e, l}\left(X^{*}\right)-\operatorname{cost}_{e}^{e l e c}\left(X^{*}\right)-\operatorname{cost}_{e}^{i m b l}\left(X^{*}\right)\right)-v_{a, l}\left(X^{*}\right)\right)= \\
\sum_{e \in A}\left(v_{e, l}\left(X_{-a}^{*}\right)-\cos t_{e}^{e l e c}\left(X_{-a}^{*}\right)-\cos t_{e}^{i m b l}\left(X_{-a}^{*}\right)\right)- \\
\sum_{e \in A}\left(v_{e, l}\left(X^{*}\right)-\operatorname{cost}_{e}^{e l e c}\left(X^{*}\right)-\operatorname{cost}_{e}^{i m b l}\left(X^{*}\right)\right)+v_{a, l}\left(X^{*}\right)
\end{gathered}
$$

Based on this equation, each agent $a$ will pay its impact on the others (i.e., its social cost) added to the cost of electricity it charged and the imbalance cost (i.e., $X_{-a}^{*}$ denotes the optimal allocation without the existence of agent $a, v_{e}\left(X^{*}\right)$ the valuation of agent $e$ based on an optimal allocation $X^{*}, \operatorname{cost}_{e}^{\text {elec }}\left(X^{*}\right)$ the electricity cost for agent $e$ based on an allocation $X^{*}$ and $\cos t_{e}^{i m b l}\left(X^{*}\right)$ the imbalance cost for $e$ based on $\left.X^{*}\right)$. The first sum contains the total values and costs for all agents, but in an allocation where $a$ does not exist. Whereas, the second sum contains the total value of all agents apart from $a$ and the costs for all agents including $a$ in an allocation where all agents participate. In all cases, the cost of electricity is fixed (per unit of energy) for all agents and acts as a reserve value for the charging station, while the imbalance cost depends on the demand profile. In contrast to the previous mechanism, this mechanism is individually rational, incentive compatible and efficient. The detailed proofs can be found in [9]. We will refer to this algorithm as VCG.

\section{EVALUATION}

In this section we evaluate our algorithms using real data regarding locations of charging stations and numbers of available plugs in Athens, Greece. ${ }^{1}$ The EV demand across the stations (and the valuations) is based on observations of traffic flow from Google maps, and discussions in related work [13]. In more detail, we evaluate the execution time and scalability (EXP1), the EV satisfaction (EXP2) and the cost for the EVs and profit for the charging stations (EXP3). We use 50 time points, where each one is assumed to be equal to 15 minutes, 10 - 130 EVs and 8 charging stations. The arrival and departure times of the EVs, the energy demand, the valuation for each energy unit and the expected demand are drawn from uniform distributions (i.e., $t_{a}^{a r r}:$ mean $=15, \sigma=15, t_{a}^{\text {dep }}:$ mean $=$ $T-t_{a}^{a r r}, b_{a}^{\text {chrg }}:$ mean $\left.=t_{a}^{d e p}-\left(t_{a}^{d e p}-t_{a}^{a r r}\right) / 2, \sigma=t_{a}^{d e p}-t_{a}^{a r r}\right) / 2$, $v_{a, l}^{\prime}:$ mean $=0.5, \sigma=0.5$ which is then multiplied by the number of energy units the agent wants to charge $v_{a, l}=v_{a, l}^{\prime} \times b_{a}^{c h r g}$ and $\operatorname{dem}_{l, t}:$ mean $=2, \sigma=1$ ). Note that the charging rate is fixed to one unit of energy per time point in all stations and that the desired energy is always able to be charged in the EV within the

${ }^{1}$ https://user.fortizo.gr/\#/portal/locations. 


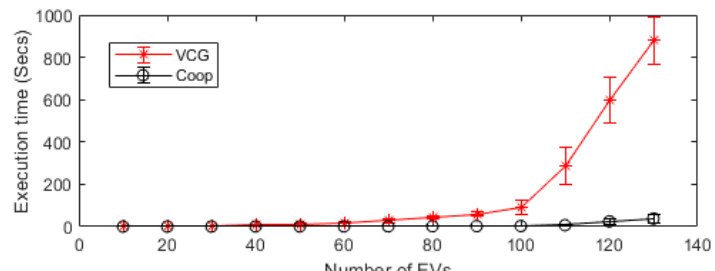

Number of EVs

Fimura 1. Fvamution timse of sll glonrithme

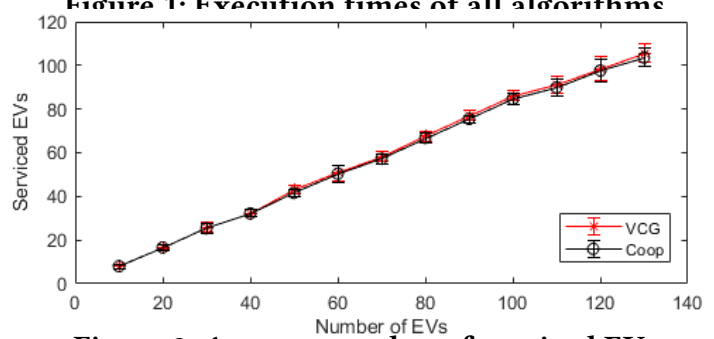

Figure 2: Average number of serviced EVs

available time window and incr $=2.5 \%$. To calculate the value for incr multiple runs of the coop mechanism were performed where the value was gradually increasing. incr $=2.5 \%$ was decided, as from this value onward the mechanism started being profitable. The results of all experiments that follow are average numbers of 100 runs.

\subsection{EXP1: Execution time and scalability}

Highly combinatorial problems such as the one we solve here are known to suffer from high execution times. Thus, it is crucial to evaluate the execution time of both algorithms and for a number of scenarios (see Figure 1). In terms of the VCG mechanism, the execution time increases quadratically $\left(R^{2}=0.898, R^{2}\right.$ is a statistical measure of how close the data are to the fitted regression line). For up to $60 \mathrm{EVs}$, the algorithm has execution times which are well under 30 seconds, but later the execution time increases rapidly and for 130 EVs it reaches 778 seconds. Overall, the second part of the objective function which contains the absolute value (i.e., the calculatio of $\cos _{l, t}^{i m b l}$ ), although it is linearized at run time, it affects the execution time a lot. As long as the Coop mechanism is concerned, the execution time also grows quadratically $\left(R^{2}=0.85\right)$. However, the rate of growth is considerably smaller compared to the equivalent times of the VCG mechanism. For example, for 130 EVs, the execution time is approximately 38 seconds. Note that the error bars in all graphs show the standard deviation of each sample.

\subsection{EXP2: EV satisfaction}

In terms of the average number of serviced EVs (Figure 2), for up to $100 \mathrm{EVs}$ on average $85 \%$ of all vehicles are charged. However, for more than $80 \mathrm{EVs}$ more of them remain uncharged, as for example for 130 EVs $81.3 \%$ of them are charged by the VCG and $79.6 \%$ by the Coop. The better performance of the VCG can be explained by the fact that the Coop does not take into consideration the valuations of the EVs in setting the prices. Thus, some EVs get higher price than their valuation and they leave without charging.

The utility of the serviced EVs (Figure 3) increases linearly with the number of EVs for both mechanisms. The rate of increase for the utility of the VCG mechanism, slows down for high numbers of EVs. This is related to the fact that when the demand for the resources

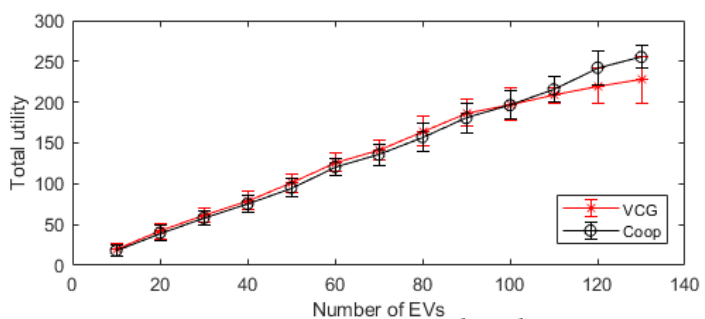

Figure 3: Average total utility

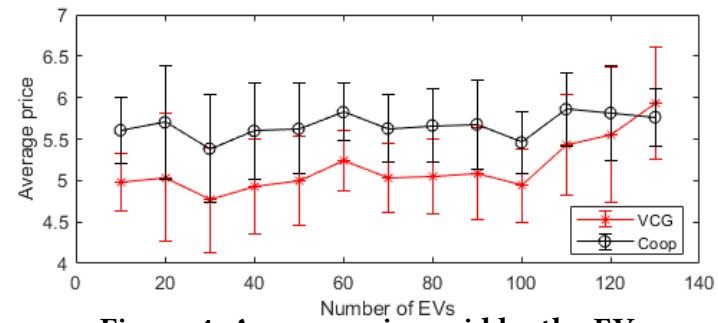

Figure 4: Average price paid by the EVs

increases a lot, this mechanism calculates high prices for the agents. Regarding the Coop, the utility for large numbers of EVs becomes higher compared to VCG. However, despite the fact that the Coop mechanism provides higher utility, it is vulnerable to manipulation in the case where EVs report their types non-truthfully. Whereas, VCG cannot be affected from such behaviour, as the EVs would have a loss any time they report anything other than their true types.

\subsection{EXP3: Price for the EVs and budget for the system}

Regarding the average price that each EV pays to the mechanism (Figure 4), the VCG calculates similar prices for up to around 100 EVs, but later they increase rapidly as the competition for the resources increases. In terms of the Coop and given that it is a fixed price mechanism, the average price mainly depends on the number of serviced EVs.

As long as the profit is concerned (Figure 5), when comparing the VCG and the Coop mechanisms the profit is initially higher for the Coop, but when the competition for the resources increases a lot (i.e., more than $100 \mathrm{EVs}$ ) the VCG achieves higher profit. However, part of the higher profit of VCG is due to the fact that this mechanism achieves higher reduction of the imbalance cost. This is so, as in VCG all EVs that are selected to charge pay prices lower than their valuations and for this reason none of them leaves the mechanism without charging. However, in the case of Coop, some EVs leave as the price to pay is higher than their valuation. For this reason the Coop mechanism pays a higher imbalance cost especially for large numbers of EVs. Thus, the profit for the Coop has only a slight increase with the number of EVs.

An interesting question is how the revenue changes with the number of charging stations. As can be seen in Figure 6, in a setting where the number of EVs is fixed to 80 and the charging stations vary from 6 to 18 , the revenue of the VCG mechanism decreases with the number of stations. This can be explained by the fact that when the number of stations is small, the competition for the resources is high and for this reason the mechanism calculates high prices. However, as the number of the stations increases, the competition 


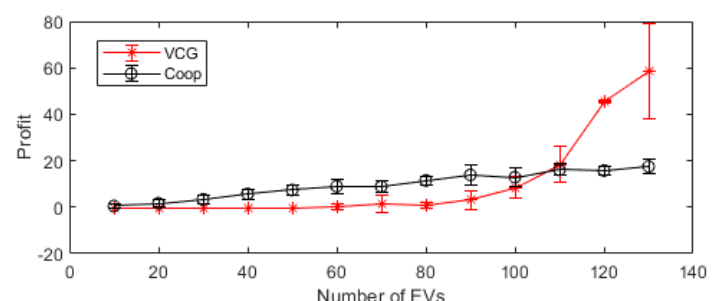

Figure 5: Total profit for the mechanism

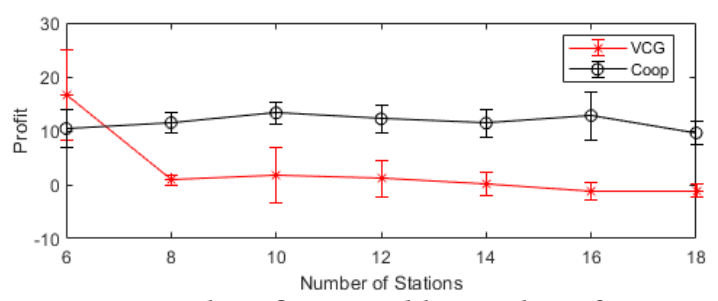

Figure 6: Total profit - Variable number of stations

decreases and so do the prices. Interestingly, for more than 14 stations, the revenue becomes negative. This can be explained by the existence of the imbalance cost: Given an equal expected demand for each station, when up to 14 of them exist, the actual demand usually overcomes the expected one. Thus, the majority of the EVs have a positive transfer and for this reason the profit for the stations is positive. However, for more than 14 stations, the expected demand is higher than the actual one, and for this reason the transfers of a number of EVs are negative (i.e., they receive a payment from the mechanism) as these EVs reduce the imbalance cost and for this reason their existence has a positive impact in the system. This finding can be used to decide on the optimal number of charging stations for an area or a city. At the same time, the Coop calculates similar prices for all cases.

\section{CONCLUSIONS AND FUTURE WORK}

In this work, we presented market-based techniques to solve the problem of scheduling EVs and allocating them to charging stations and we considered two approaches. In the first approach we used a fixed price mechanism, while in the second approach, we used the well known VCG mechanism where truthtelling is the dominant strategy. We evaluated our algorithms in a realistic setting and we observed that both have good scalability. Moreover, we observed that the VCG mechanism leads to higher revenue for the stations and lower utility for the EVs in cases where the stations are highly congested. However, it is proven to not be vulnerable to agents' strategic behaviour.

As far as future work is concerned, we aim to apply online mechanism design techniques for the same problem, while we also want to add V2G and V2V energy transfer so as to use the EVs' batteries as storage devices and increase energy utilization and customer satisfaction [7]. Moreover, we aim to use queuing theory in order to add the ability for the EVs to queue in the charging stations. Finally, it is crucial to investigate machine learning techniques in order to achieve good prediction in future demand and minimise the imbalance cost.

\section{ACKNOWLEDGMENT REFERENCES}

[1] U.S. Energy Information Administration. 2019. Annual Energy Outlook 2019. Technical Report.

[2] Edward H Clarke. 1971. Multipart pricing of public goods. Public choice 11, 1 (1971), 17-33.

[3] J.M. Foster and M.C. Caramanis. 2013. Optimal Power Market Participation of Plug-In Electric Vehicles Pooled by Distribution Feeder. Power Systems, IEEE Transactions on 28, 3 (Aug 2013), 2065-2076. https://doi.org/10.1109/TPWRS. 2012.2232682

[4] Enrico H. Gerding, Valentin Robu, Sebastian Stein, David C. Parkes, Alex Rogers, and Nicholas R. Jennings. 2011. Online Mechanism Design for Electric Vehicle Charging. In AAMAS-11 - Volume 2 (Taipei, Taiwan). International Foundation for Autonomous Agents and Multiagent Systems, Richland, SC, 811-818.

[5] A. Ghosh and V. Aggarwal. 2017. Control of Charging of Electric Vehicles through Menu-Based Pricing. IEEE Transactions on Smart Grid PP, 99 (2017), 1-1. https://doi.org/10.1109/TSG.2017.2698830

[6] Theodore Groves. 1973. Incentives in teams. Econometrica: Journal of the Econometric Society (1973), 617-631.

[7] A. Koufakis, E. S. Rigas, N. Bassiliades, and S. D. Ramchurn. 2020. Offline and Online Electric Vehicle Charging Scheduling With V2V Energy Transfer. IEEE Transactions on Intelligent Transportation Systems 21, 5 (2020), 2128-2138.

[8] W. Liu, Y. Gong, W. Chen, Z. Liu, H. Wang, and J. Zhang. 2019. Coordinated Charging Scheduling of Electric Vehicles: A Mixed-Variable Differential Evolution Approach. IEEE Transactions on Intelligent Transportation Systems (2019), 1-16.

[9] E. S. Rigas, E. Gerding, S. Stein, S. D. Ramchurn, and N. Bassiliaded. 2020. Mechanism Design for Efficient Online and Offline Allocation of Electric Vehicles to Charging Stations. In arXiv:2007.09715.

[10] Emmanouil S. Rigas, Sarvapali D. Ramchurn, and Nick Bassiliades. 2015. Managing Electric Vehicles in the Smart Grid Using Artificial Intelligence: A Survey. Intelligent Transportation Systems, IEEE Transactions on 16, 4 (Aug 2015), 16191635. https://doi.org/10.1109/TITS.2014.2376873

[11] Emmanouil S. Rigas, Sarvapali D. Ramchurn, Nick Bassiliades, and George Koutitas. 2013. Congestion management for urban EV charging systems. In Smart Grid Communications (SmartGridComm), 2013 IEEE International Conference on. 121-126. https://doi.org/10.1109/SmartGridComm.2013.6687944

[12] Andreas Seitaridis, Emmanouil S. Rigas, Nick Bassiliades, and Sarvapali D. Ramchurn. 2020. An agent-based negotiation scheme for the distribution of electric vehicles across a set of charging stations. Simulation Modelling Practice and Theory 100 (2020), 102040. https://doi.org/10.1016/j.simpat.2019.102040

[13] A Spiliopoulou, M Kontorinaki, M Papageorgiou, and P Kopelias. 2014. Macroscopic traffic flow model validation at congested freeway off-ramp areas. Transportation Research Part C: Emerging Technologies 41 (2014), 18-29.

[14] Sebastian Stein, Enrico Gerding, Valentin Robu, and Nicholas R. Jennings. 2012. A Model-based Online Mechanism with Pre-commitment and Its Application to Electric Vehicle Charging. In Proceedings of the 11th International Conference on Autonomous Agents and Multiagent Systems - Volume 2 (Valencia, Spain) (AAMAS '12). International Foundation for Autonomous Agents and Multiagent Systems, Richland, SC, 669-676.

[15] William Vickrey. 1961. Counterspeculation, auctions, and competitive sealed tenders. The fournal of finance 16, 1 (1961), 8-37.

[16] Michael Wooldridge. 2009. An introduction to multiagent systems. John Wiley \& Sons. 